\title{
Fine Structure of the Rydberg Blockade Zone
}

\author{
Yurii V. Dumin* \\ Moscow State University, GAISh, Universitetski pr. 13, 119992, Moscow, Russia and \\ Space Research Institute (IKI) of Russian Academy of Sciences, \\ Profsoyuznaya str. 84/32, 117997, Moscow, Russia
}

(Dated: December 30, 2013)

\begin{abstract}
A spatial structure of the zone blocked by the dipolar electric field of a Rydberg atom is calculated taking into account a possibility of excitation to the states with neighboring values of the principal quantum number. As a result, it was found that the blocked zone represents a number of co-centric spherical shells rather than a solid ball, and the respective pair correlation function should have additional maxima at small interparticle distances.
\end{abstract}

PACS numbers: 32.80.Ee, 32.60.+i

The concept of Rydberg blockade of the ultracold gas began to be widely discussed in atomic physics since the early 2000's [1], and a few years later a possibility of this effect was verified experimentally [2-4]. In the resent time, the idea of Rydberg blockade is used in a lot of intriguing phenomena, such as a highly efficient entanglement between the light and atoms [5, 6], observation of the spatially ordered structures in a Rydberg gas [7], realization of strong interaction between photons [8, 9] and, particularly, creation of the photon pairs [10, 11], etc.

The basic mechanism of the Rydberg blockade can be illustrated in Fig. 1. It is assumed here that Rydberg atom with principal quantum number $n$ located in the origin of coordinates $(r=0)$ produces an electric field which disturbs the energy levels of neighboring atoms. For example, in the simplest case of linear (with respect to the field) Stark effect a highly-excited electron state $|n\rangle$ will be split into a symmetric series of sublevels, characterized by the so-called parabolic quantum numbers [12, 13]. The degree of splitting evidently increases as we approach the origin of coordinates, which is shown in figure by a series of diverging curves. Then, when a disturbed sublevel goes away from the characteristic bandwidth of the exciting radiation $\Delta E$ (shown by a pair of dotted horizontal lines), the excitation will no longer be possible. Therefore, the "excitation zone" of the specified sublevel should take place at $r \geqslant R_{\mathrm{b}}^{(n)}$ (which is marked by the thick strip near the horizontal axis), while at $r<R_{\mathrm{b}}^{(n)}$ the Rydberg blockade develops [18].

Unfortunately, the outlined standard picture of Rydberg blockade does not take into account a presence of other energy levels with close values of the principal quantum number, e.g., $|n-1\rangle$ and $|n+1\rangle$. In fact, these energy levels experience a similar splitting by the external electric field, so that the respective sublevels can enter the energy band of the exciting radiation $\Delta E$ at the sufficiently small distances $r$. Namely, as illustrated in Fig. 1. the most disturbed sublevel from the series $|n-1\rangle$ enters the band $\Delta E$ at the distance $R_{\mathrm{u}}^{(n-1)}$, so that the Rydberg excitation should be unblocked (which is denoted by

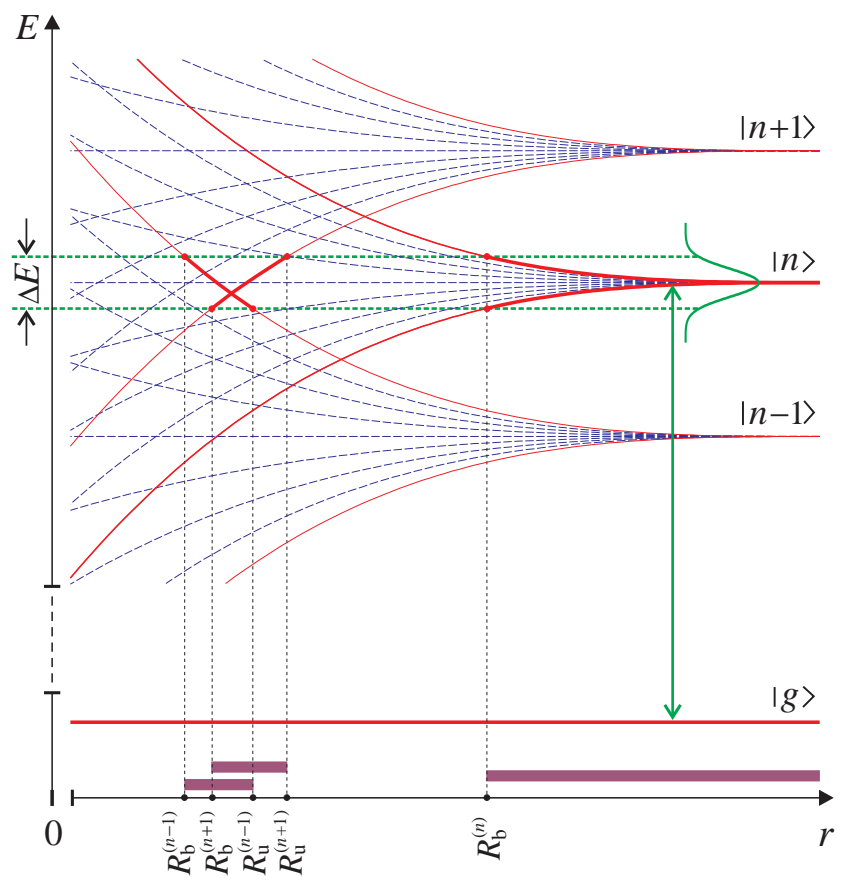

FIG. 1: Splitting the energy levels of a neighboring atom by the electric field of the central Rydberg-excited atom. Sublevels with maximal splitting are shown by solid (red) curves; and other sublevels, by broken (blue) curves. Rydberg excitation is allowed in the thick segments of energy curves, located within the dotted (green) lines, designating a characteristic bandwidth of the exciting radiation. The corresponding intervals of radius $\left[R_{\mathrm{b}}^{(n-1)}, R_{\mathrm{u}}^{(n-1)}\right],\left[R_{\mathrm{b}}^{(n+1)}, R_{\mathrm{u}}^{(n+1)}\right]$, and $\left[R_{\mathrm{b}}^{(n)},+\infty\right]$, where Rydberg excitation can take place, are marked by thick (violet) strips near the horizontal axis.

subscript ' $\mathrm{u}$ '). Next, at the smaller distance $R_{\mathrm{b}}^{(n-1)}$ this excitation will be blocked again, since the corresponding energy level comes out of the irradiation bandwidth. The same effect should take place evidently for the split sublevels of the state $|n+1\rangle$, unblocking the Rydberg excitation in the range of distances $R_{\mathrm{u}}^{(n+1)}$ and $R_{\mathrm{b}}^{(n+1)}$.

As a result, along with the commonly considered ex- 
citation zone $r \geqslant R_{\mathrm{b}}^{(n)}$, the additional excitation zones $\left[R_{\mathrm{b}}^{(n-1)}, R_{\mathrm{u}}^{(n-1)}\right], \quad\left[R_{\mathrm{b}}^{(n+1)}, R_{\mathrm{u}}^{(n+1)}\right], \quad\left[R_{\mathrm{b}}^{(n-2)}, R_{\mathrm{u}}^{(n-2)}\right]$, $\left[R_{\mathrm{b}}^{(n+2)}, R_{\mathrm{u}}^{(n+2)}\right]$, etc. will emerge (thick strips near the left-hand part of the horizontal axis in figure). Therefore, domain of the Rydberg blockade (corresponding to the radii beyond the above-mentioned strips) will represent a number of co-centric spherical shells rather than a solid ball. It is quite surprising that possibility of emergence of this fine structure was not taken into account before.

Let us perform some quantitative estimates. For simplicity, we shall consider a dipolar electric field by the central Rydberg atom averaged over orientation of the dipole:

$$
\mathcal{E}(r)=\frac{C_{3} e a_{0} n^{2}}{r^{3}},
$$

where $C_{3}$ is a numerical coefficient on the order of unity, $n$ is the principal quantum number, $a_{0}=\hbar^{2} /\left(m e^{2}\right)$ is Bohr radius, $\hbar$ is Planck constant, $e$ and $m$ are the electron charge and mass. In the atomic units, marked by tildes, this formula can be rewritten as

$$
\tilde{\mathcal{E}}(\tilde{r})=\frac{C_{3} n^{2}}{\tilde{r}^{3}}
$$

where $r=a_{0} \tilde{r}, \mathcal{E}=\mathcal{E}^{*} \tilde{\mathcal{E}}$, and $\mathcal{E}^{*}=m^{2} e^{5} / \hbar^{4}$.

Next, we shall assume that this electric field is approximately constant at the scale of the neighboring atom, whose energy levels are split. Such an approximation was often used in the early works on Rydberg blockade. In fact, as follows from the modern treatments, nonuniformity of the electric field is essential; so that the resulting interaction between the atoms should be of the Van der Waals type, whose energy is proportional to $r^{-6}$. Unfortunately, treating the perturbations of energy levels by the nonuniform field represents a much more difficult task, which requires a separate paper. So, we shall perform our estimates here ignoring the nonuniformity.

Then, under the above-mentioned assumptions, splitting the energy levels is given by the well-known formula for the linear Stark effect [12, 13]:

$$
\tilde{E}_{n n_{1} n_{2}}=-\frac{1}{2 n^{2}}+\frac{3}{2}\left(n_{1}-n_{2}\right) n \tilde{\mathcal{E}},
$$

where $E=E^{*} \tilde{E}, E^{*}=m e^{4} / \hbar^{2}, n_{1}$ and $n_{2}$ are the so-called parabolic quantum numbers, which are nonnegative integers, satisfying the condition:

$$
n_{1}+n_{2}+|m|+1=n
$$

where $m$ is the magnetic quantum number.

In particular, at $m=0$ the difference of parabolic quantum numbers can take the following values: $n_{1}-n_{2}=$ $n-1, n-3, \ldots,-n+1$; at $m=1, n_{1}-n_{2}=n-2, n-$ $4, \ldots,-n+2 ;$ and so on.
Let us take, for example, $m=0$, which closely resembles the real experiments on Rydberg blockade [19]. Then, expression (3) can be rewritten as

$$
\tilde{E}_{n k}=\frac{1}{2}\left\{-\frac{1}{n^{2}}+3 k n \tilde{\mathcal{E}}\right\},
$$

where we denoted for conciseness

$$
k \equiv n_{1}-n_{2}=n-1, n-3, \ldots,-n+1
$$

Since Rydberg blockade is defined by the behavior of the most disturbed energy levels, we shall consider further the sublevels with $k= \pm(n-1)$.

As is seen in Fig. 1, blockade of the basic state $|n\rangle$ (i.e., the state with the same principal quantum number as for the central Rydberg atom) develops under condition:

$$
\tilde{E}_{n, \pm(n-1)}\left(\tilde{\mathcal{E}}_{\mathrm{b}}^{(n)}\right)=\tilde{E}_{n, \pm(n-1)}(0) \pm \frac{1}{2} \Delta \tilde{E}
$$

where $\tilde{\mathcal{E}}_{\mathrm{b}}^{(n)}=\tilde{\mathcal{E}}\left(\tilde{R}_{\mathrm{b}}^{(n)}\right)$, and $\Delta \tilde{E}$ is the characteristic bandwidth of the exciting irradiation. Substitution of (5) to (17) results in

$$
3 n^{2} \tilde{\mathcal{E}}_{\mathrm{b}}^{(n)}=\Delta \tilde{E}
$$

where we have ignored the corrections of order $n^{-1}$.

Next, let us consider formation of the additional excitation zone $\left[R_{\mathrm{b}}^{(n-1)}, R_{\mathrm{u}}^{(n-1)}\right]$ due to the entrance of a strongly disturbed sublevel of the low-lying state $|n-1\rangle$ into the band of exciting irradiation. As follows from (6), the corresponding sublevel should have $k=(n-1)-1=$ $n-2$. Therefore, the required condition can be formulated as

$$
\tilde{E}_{n-1, n-2}\left(\tilde{\mathcal{E}}_{\mathrm{u}, \mathrm{b}}^{(n-1)}\right)=\tilde{E}_{n}(0) \mp \frac{1}{2} \Delta \tilde{E}
$$

where minus sign refers to the situation when the perturbed sublevel passes the lower boundary of the excitation band (i.e., the Rydberg excitation is unblocked), and plus sign refers to the situation when this sublevel passes the upper boundary (i.e., the Rydberg excitation is blocked again). Substitution of (5) to (9) results in

$$
3 n^{5} \tilde{\mathcal{E}}_{\mathrm{u}, \mathrm{b}}^{(n-1)}=2 \mp n^{3} \Delta \tilde{E},
$$

where terms of the order $n^{-1}$ were ignored again.

At last, combining expressions (8) and (10) we arrive at the following equality:

$$
3 n^{5}\left[\tilde{\mathcal{E}}_{\mathrm{u}, \mathrm{b}}^{(n-1)} \pm \tilde{\mathcal{E}}_{\mathrm{b}}^{(n)}\right]=2,
$$

which gives a relation between the electric field strength in the main and additional zones of Rydberg excitation.

Substitution of formula (2) for the average dipolar electric field produced by the central Rydberg atom into (11) 
enables us to find the boundaries of the additional excitation zone:

$$
\tilde{R}_{\mathrm{u}, \mathrm{b}}^{(n-1)}=\left(\frac{3 C_{3} n^{7}}{2}\right)^{1 / 3}\left\{1 \mp \frac{3 C_{3} n^{7}}{2\left(\tilde{R}_{\mathrm{b}}^{(n)}\right)^{3}}\right\}^{-1 / 3}
$$

or, if the second term is small as compared to the first one,

$$
\tilde{R}_{\mathrm{u}, \mathrm{b}}^{(n-1)} \approx\left(\frac{3 C_{3} n^{7}}{2}\right)^{1 / 3}\left\{1 \pm \frac{C_{3} n^{7}}{2\left(\tilde{R}_{\mathrm{b}}^{(n)}\right)^{3}}\right\} .
$$

A similar analysis can be performed for split sublevels of the higher-lying state $|n+1\rangle$. The most disturbed sublevel in this case will have $k=-(n+1)+1=-n$. Although the intermediate formulas are somewhat different, the final result in the first approximation turns out to be exactly the same as (12) and (13). (However, expressions for $\tilde{R}_{\mathrm{u}, \mathrm{b}}^{(n-1)}$ and $\tilde{R}_{\mathrm{u}, \mathrm{b}}^{(n+1)}$ will be slightly different if terms of the order $n^{-1}$ are taken into account. This fact is schematically shown in Fig. 1.)

Therefore, as follows from the above-written formulas, center of the additional excitation zone is located at the distance

$$
\tilde{R}_{\mathrm{c}}^{(n-1)}=\left(\frac{3 C_{3} n^{7}}{2}\right)^{1 / 3}
$$

and its characteristic width equals

$$
\Delta \tilde{R}^{(n-1)}=\left(\frac{3}{2}\right)^{1 / 3} \frac{C_{3}^{4 / 3} n^{28 / 3}}{\left(\tilde{R}_{\mathrm{b}}^{(n)}\right)^{3}} .
$$

As should be expected, position of the additional zone is function of only the principal quantum number (i.e., location of the energy level), while its width depends also on the radius of the main blockade zone. Really, decrease in $\tilde{R}_{\mathrm{b}}^{(n)}$ implies a broader excitation band $\Delta \tilde{E}$ and, therefore, a wider additional excitation zone.

Let us perform the numerical estimates for a particular experiment, e.g. 7], where $n=43$ and $R_{\mathrm{b}}^{(n)}=4 \mu \mathrm{m}$ $\left(\tilde{R}_{\mathrm{b}}^{(n)}=7.6 \times 10^{4}\right)$. Then, assuming that the numerical factor $3 C_{3} / 2$ is about unity, we get $\tilde{R}_{\mathrm{c}}^{(n-1)} \approx 6.5 \times 10^{3}$ or $R_{\mathrm{c}}^{(n-1)} \approx 0.34 \mu \mathrm{m}$. In fact, the pair correlation function of Rydberg atoms presented in Fig. 3a of paper [7] really has a strong unexpected maximum at the radii $r \lesssim 0.5 \mu \mathrm{m}$, i.e., well inside the expected Rydberg blockade zone. However, this maximum was attributed by the authors to the imperfection of the detection procedures. On the other hand, as follows from the above-written estimates, this extra peak could have a deep physical meaning. Namely, it might be caused just by the excitation of Rydberg states with neighboring principal quantum numbers $n-1, n+1$, etc.
As regards a characteristic width of the additional excitation zone, formula (15) gives $\Delta \tilde{R}^{(n-1)} \sim 5 \div 10$, i.e., only about a typical size of the ground-state atom. However, it should be kept in mind that this excitation zone is actually formed by a very large number $\left(\sim n^{2}\right)$ of the split sublevels, which sequentially enter and leave the energy band $\Delta E$ in Fig. 1. So, the overall width of the additional excitation zone should be substantial.

In conclusion, let us mention that the effect under consideration may be important also in the experiments on the formation of ultracold plasmas from Rydberg gases (e.g., [14 16]). Namely, one of the well-known phenomena is a spontaneous ionization of the neutral Rydberg gas, which usually requires a number of seed electrons for the ionization avalanche to develop [17]. It can be conjectured that just the emergence of the additional excitation zones at the small interparticle separation and the resulting reconfiguration of the electric field pattern lead to the inherent instability of the system and the release of free electrons.

In summary, we have shown that the Rydberg blockade zone should represent a number of co-centric shells rather than a solid sphere, as it was commonly assumed before. In fact, the presence of such inner shells, manifesting themselves as an additional peak in the pair correlation function at small distances, was already seen in some experiments. Emergence of the additional excitation zones can, firstly, reduce an overall efficiency of the Rydberg blockade and, secondly, result in the release of some number of free electrons. Such effects should be taken into account in the design and interpretation of future experiments.

I am grateful to Prof. A. Buchleitner for valuable discussions, consultations, and advises.

* dumin@yahoo.com,dumin@sai.msu.ru

[1] M. Lukin, M. Fleischhauer, R. Cote, L. Duan, D. Jaksch, J. Cirac, and P. Zoller, Phys. Rev. Lett. 87, 037901 (2001).

[2] D. Tong, S. Farooqi, J. Stanojevic, S. Krishnan, Y. Zhang, R. Côté, E. Eyler, and P. Gould, Phys. Rev. Lett. 93, 063001 (2004).

[3] K. Singer, M. Reetz-Lamour, T. Amthor, L. Gustavo Marcassa, and M. Weidemüller, Phys. Rev. Lett. 93, 163001 (2004).

[4] M. Weidemüller, Nature Physics 5, 91 (2009).

[5] L. Li, Y. Dudin, and A. Kuzmich, Nature (London) 498, 466 (2013).

[6] M. Weidemüller, Nature (London) 498, 438 (2013).

[7] P. Schauß, M. Cheneau, M. Endres, T. Fukuhara, S. Hild, A. Omran, T. Pohl, C. Gross, S. Kuhr, and I. Bloch, Nature (London) 491, 87 (2012).

[8] T. Peyronel, O. Firstenberg, Q.-Y. Liang, S. Hofferberth, A. Gorshkov, T. Pohl, M. Lukin, and V. Vuletić, Nature (London) 488, 57 (2012).

[9] T. Walker, Nature (London) 488, 39 (2012). 
[10] O. Firstenberg, T. Peyronel, Q.-Y. Liang, A. Gorshkov, M. Lukin, and V. Vuletić, Nature (London) 502, 71 (2013).

[11] S. Bose, Nature (London) 502, 40 (2013).

[12] T. Gallagher, Rydberg Atoms (Cambridge Univ. Press, Cambridge, UK, 1994).

[13] B. Yavorsky and A. Detlaf, Handbook of Physics (Mir, Moscow, 1980), 3rd ed.

[14] P. Gould and E. Eyler, Phys. World 14(3), 19 (2001).

[15] S. Bergeson and T. Killian, Phys. World 16(2), 37 (2003).

[16] Y. Dumin, Plas. Phys. Rep. 37, 858 (2011).
[17] M. Robert-de Saint-Vincent, C. Hofmann, H. Schempp, G. Günter, S. Whitlock, and M. Weidemüller, Phys. Rev. Lett. 110, 045004 (2013).

[18] In fact, as is seen in Fig. 1] the excitation of different sublevels will be blocked at various radii $r$. However, for the sake of definiteness, we shall characterize the overall Rydberg blockade by the behaviour of the most disturbed sublevel.

[19] Almost all experiments performed by now employed the energy levels with $|m| \leqslant 2 \ll n$, where $n$ is the principal quantum number of the Rydberg state. 